\title{
Broadband Azimuthal Processing with Diffraction Imaging
}

\author{
A. M. Popovici, I. Sturzu, N. Tanushev
}

Copyright 2019, SBGf - Sociedade Brasileira de Geofísica

This paper was prepared for presentation during the $16^{\text {th }}$ International Congress of the Brazilian Geophysical Society held in Rio de Janeiro, Brazil, 19-22 August 2019.

Contents of this paper were reviewed by the Technical Committee of the $16^{\text {th }}$ International Congress of the Brazilian Geophysical Society and do not necessarily represent any position of the SBGf, its officers or members. Electronic reproduction or storage of any part of this paper for commercial purposes without the written consent of the Brazilian Geophysical Society is prohibited.

\section{Abstract}

Diffraction Imaging (DI) is a high-resolution imaging technology designed to image and identify in very fine detail the small scale fractures in shale and carbonate reservoirs that form areas of increased natural fracture density. Diffraction Imaging provides a separate 3D (stack), 4D (angle gathers) or 5D (angle and azimuth gathers) image of discontinuities, or objects which are small compared to the wavelength of seismic waves such as fault edges, small scale faults, fractured zones, pinchouts, reef edges, channel edges, salt flanks, reflector unconformities, injectites, fluid fronts, caves and karst, in general any small scattering objects. Diffraction imaging is the direct response to subsurface discontinuities and is in most cases obtained from pre-stack, pre-migration data rather than post-stack, post-migration images. The diffractions volume can be used as a complement to the structural images produced by reflection imaging (Khaidukov et al., 2004; Taner et al., 2006; Moser and Howard, 2008; Koren et al., 2010; Dell and Gajewski, 2011).

Standard approaches to obtain high-resolution information, such as coherency analysis and structureoriented filters, derive attributes from stacked, migrated images. Diffraction imaging in comparison, acts on the pre-stack data, and as a result can provide both angular and azimuthal amplitude information in addition to the high resolution structural information.

An important point to note is that a true diffraction image is not optimally obtained by post-processing a traditional seismic image, even if the seismic image is obtained by an algorithm that does not suppress diffractions. While diffractors will appear in the image, usually in the form of discontinuities, they have much lower amplitudes than reflecting structures, by a factor of 10 or 100 , depending on acquisition geometry and fold. By imaging diffractors using the pre-stack data, the diffractor amplitude can be enhanced while the specular reflections can be attenuated. Seismic methods are generally limited in their resolving power to about one half of the dominant wavelength at the target. When the sand or shale layers are thinner than half of the wavelength, tuning and multiple-reverberation effects make the stratigraphic interpretation of the images difficult and unreliable.

We show that operating in a migration framework on prestack data, using procedures which complement those used to enhance specular reflections, allows us to obtain higher resolution information, which is lost in conventional procedures. An efficient way to obtain diffraction images is to first separate the migration events according to the value of specularity angle and azimuth, in a similar way to offset or angle azimuth gathers; diffraction images are produced subsequently using post-processing procedures. The high-resolution potential is demonstrated by several case histories in carbonate reservoirs and unconventional shales, which show much more detail than conventional depth migration or coherence. In addition the variation of the diffractions amplitudes with azimuth gives information about the direction of the local stress field, thus allowing reservoir and drilling engineers to optimize the horizontal well trajectories.

Project experience has shown that it is not uncommon for two nearby wells to achieve substantially different levels of production despite extremely similar geology and appearance in reflection-based seismic images. If Diffraction Imaging allows us to identify and classify naturally-occurring fractures, we will gain valuable information about the existing stress field, and the suitability of a given location for fracturing - before drilling.

\section{Introduction}

The unconventional shales drilling technology was spearheaded in the mid 2000s by small companies with expert drilling engineers, without much focus on seismic imaging, reservoir engineering, or field optimization. The emphasis was on drilling cost and on recovering the drilling cost for each well through individual well production. As larger companies with reservoir characterization and reservoir optimization teams got involved, the emphasis changed from the cost of individual wells to field optimization. It was noticed that wells placed very close using a grid pattern could have very different oil and gas production. Figure 1 shows a map view of the grid pattern used to develop an actual unconventional shales field. The wells are placed on a chess board grid, without regard to the underlying geology. The hypothesis is that the structure is very simple and therefore the production should be uniform. In reality the production is not uniform and two wells placed very close by can have very different production.

Figure 2 shows such an example in an unconventional shale play, two very close wells $A$ and $B$, penetrating what seems to be a very simple layer in a very un-complicated, sediment layers geology. One of the wells produces twice the amount of the other. After normalizing for identical engineering details and hydraulic fracturing parameters (frac design) the explanation lies in different geologic conditions for each of the wells. 


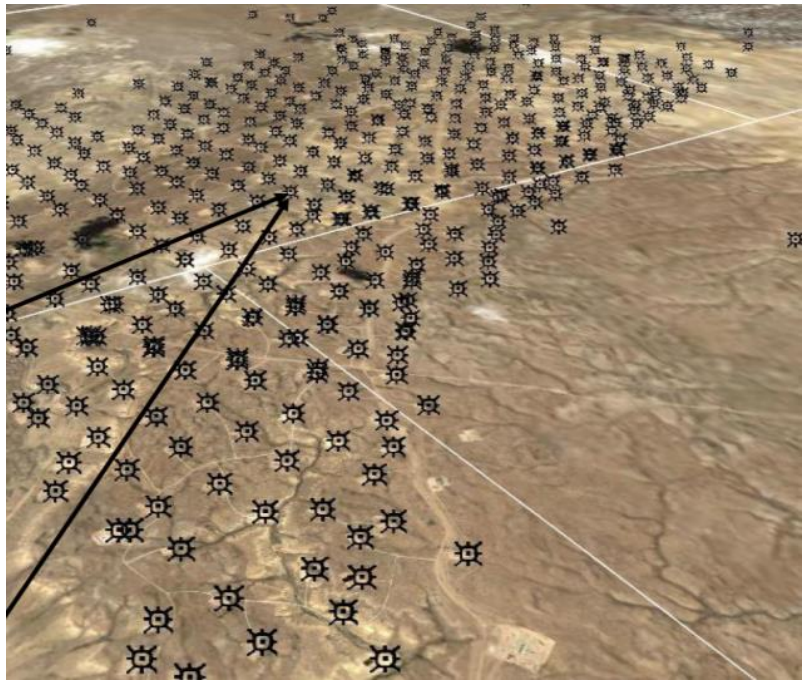

Figure 1: Oil well placement in an unconventional shales field.

We notice that the well on the left which has higher production, seems to penetrate an area with higher diffraction imaging amplitudes which correlate with areas of increased natural fracturing. Maybe the reason for higher production is that well A perforates an area with increased gas production due to the natural fractures.

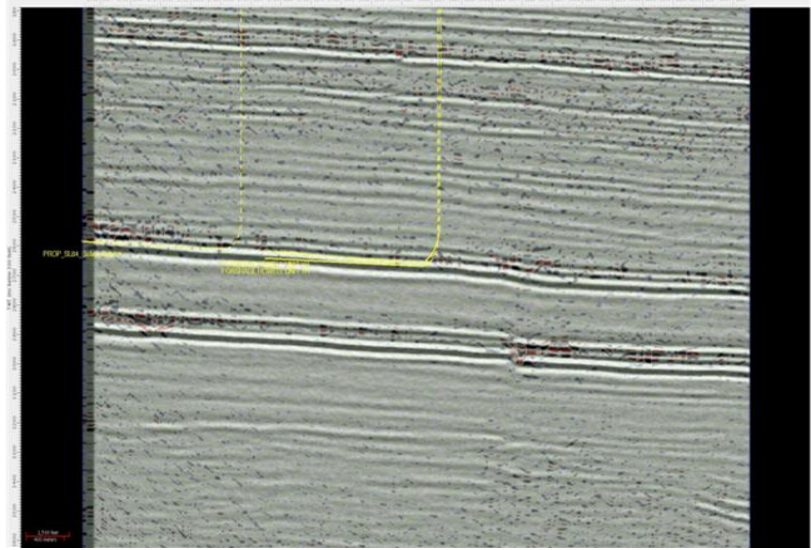

Figure 2: Two very similar wells drilled through an unconventional shales play. The grey scale shows the geological structure while the red-to-blue scale shows the diffraction imaging amplitudes overlayed. The well on the left produces twice the amount of oil than the well on the right. The geology and sediment layers structure seems very similar. The amount of natural fracturing along each horizontal well seems to be very different based on diffraction imaging amplitudes.

Accounting for the geologic conditions becomes an important factor in an optimization problem. Placing the wells too close wastes drilling money. Placing the wells too far leaves resources in the ground. Given the fact that a well in unconventional shales may cost $\$ 6-12$ million, optimizing the well placement of hundreds of wells in a field becomes a very large cost savings and environmental protection technology. One measure of natural fracturing which correlates with higher production rates can be obtained using Diffraction Imaging technology, designed to image with super-resolution small scale discontinuities in the sediment layers using standard 3-D seismic surface data. Since diffractors are, by definition, smaller than the wavelength of seismic waves, diffraction imaging provides super-resolution information.

In Figure 3 we see the difference in detail between a depth slice through a Kirchhoff migrated data volume in the Eagle Ford, and the same depth slice through a 3D diffraction imaging volume. The diffraction imaging volume offers higher resolution details of discontinuities, such as the natural fractures occurring in rocks.
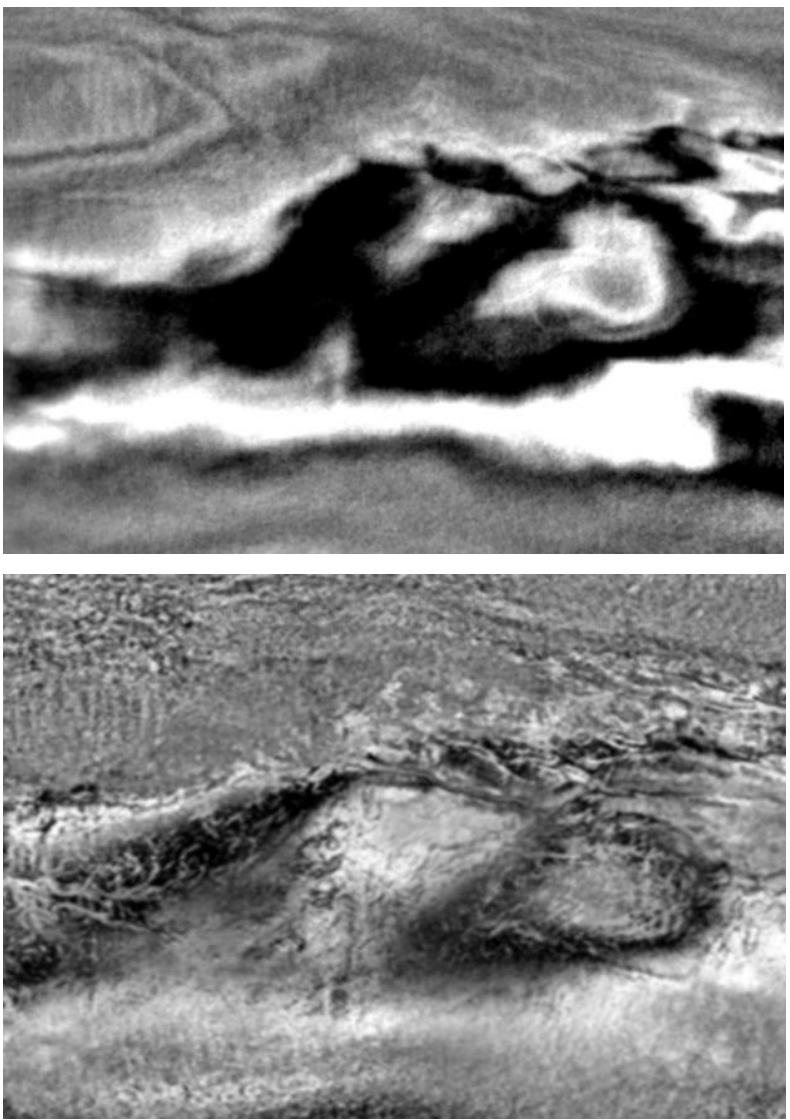

Figure 3: Comparison between an Eagle Ford Kirchhoff depth slice on the left and a diffraction depth slice of the same data on the right. The diffraction depth slice offers higher resolution details of discontinuities, such as the natural fractures occurring in rocks.

\section{Method and Examples}

Diffractions are the seismic response of small elements (or diffractors) in the subsurface of the earth, such as small scale faults, near surface scattering objects and in general all objects which are small compared to the wavelength of seismic waves. Diffraction imaging is simply the process of using diffractions to determine the locations of the small subsurface elements that produced them. Since diffractors are, by definition, smaller than the wavelength of seismic waves, diffraction imaging provides super-resolution information, which consists of image details that are beyond the classical Rayleigh limit of half a seismic wavelength. The importance of diffractions in high-resolution structural imaging has been emphasized 
in many recent publications (Tanner et al. 2006, Fomel et al. 2006, Khaidukov et al. 2004, Moser and Howard 2008, Moser 2009, Popovici et al., 2014), however, diffraction imaging is still not a widely used tool in seismic interpretation. In fact, most of the algorithms that are used to process seismic data enhance reflections and suppress diffracted energy. The goal of diffraction imaging is not to replace these traditional algorithms, but rather to provide interpreters with an additional image to fill in the small, but potentially crucial, structural details.

An important point to note is that a true diffraction image is not optimally obtained by post-processing of a traditional seismic image, even if the seismic image is obtained by an algorithm that does not suppress diffractions. While diffractors will appear in the image, usually in the form of discontinuities, they have much lower amplitudes than reflecting structures. By imaging diffractors using the pre-stack data, the diffractor amplitude can be enhanced while the specular reflections can be attenuated. Furthermore, and more importantly, discontinuities in the seismic image can appear for a variety of reasons other than diffractions, including small errors in the velocity model of the earth that was used to obtain the image.

Several techniques for diffraction imaging have been proposed (Khaidukov et al. 2004, Tanner et al. 2006, Moser and Howard 2008, Moser 2009). They fall into two categories. In the first category are methods that separate the seismic data into two parts, one that contains the wave energy from reflections and the other that contains the wave energy from diffractions. Each component is used to provide an image through traditional seismic imaging methods. We have to keep in mind that there is no clear distinction between "reflection waves" and "diffraction waves." By Huygens' principle a reflector can be represented by a series of point diffractors that are positioned on its surface. In the second category are methods that do not separate the input seismic data, but rather use a different image forming technique that suppresses reflecting surfaces in the image (Moser and Howard 2008, Moser 2009). We will focus on the second category of methods, specifically on the method of Moser and Howard, which can be expressed as a reflection suppressing kernel for Kirchhoff migration.

A conventional full wave Kirchhoff migration forms a seismic image as

$$
V_{\text {kirch }}(x)=\sum_{s, r} \int U(t, s, r) \delta\left(t-t_{d}(s, x, r)\right) d t,(1)
$$

where, $\delta$ is the Dirac delta function, the sum is over all source and receiver pairs $(s, r), U(t, s, r)$ is the seismic data and $t_{d}(s, x, r)$ is the stacking traveltime trajectory given by the traveltime from the source to the image point $x$ and back to the receiver, $t_{d}(s, x, r)=T(s, x)+T(x, r)$. For a sufficiently dense grid covering the source-receiver acquisition, the traveltimes $T(s, x)$ and $T(x, r)$ are pre-computed by ray tracing in the velocity model, and stored on disk as travel-time tables for subsequent use in forming the image (1).

The idea behind Kirchhoff diffraction imaging is to modify the image to be given by

$W_{\text {kirch }}(x)=\sum_{s, r} \int_{w} w(s, x, r) U(t, s, r) \delta\left(t-t_{d}(s, x, r)\right) d t$,

where all of the quantities are the same as before, except for the addition of the weight function $w(s, x, r)$. This weight is used to suppress reflections and is obtained by the following steps. First, using standard Kirchhoff migration we find the seismic image $V_{\text {kirch }}(x)$ by equation (1). This image will include both reflections and diffractions, but as mentioned before, the reflections are the most dominant part of the image. The second step is to analyze the structures in the Kirchhoff image and determine the normal vector $\hat{n}(x)$ to these structures at each image point $x$. With this information we define the weight function by

$w(s, x, r)=1-\left|\frac{\hat{n} \cdot\left(p^{s}+p^{r}\right)}{\left\|p^{s}+p^{r}\right\|}\right|$,

where $p^{s}(x)=\nabla_{x} T(s, x)$ and $p^{r}(x)=\nabla_{x} T(x, r)$ are the gradients of the traveltimes from the source to the image point and from the image point to the receiver respectively. The logic behind this weight function is that the vector $\left(p^{s}+p^{r}\right) /\left\|p^{s}+p^{r}\right\|$ is aligned with the direction of wave propagation. At a reflector, the wave direction is collinear with the normal vector to the reflector, thus the weight function will be nearly zero. At a diffraction point, since the seismic waves propagate in all directions, the weight function will not be zero, at least for a large part of the wave propagation directions.

Principal Componenet Analysis (PCA) also named Singular Value Decomposition (SVD) is a multivariate statistical technique that can be successfully applied only if certain conditions are satisfied. Particularly, it is essential that the multivariate statistical samples share a certain amount of similarity. The maximum number of principal components that can be identified is limited by the number of variables. If the statistical samples are too dissimilar, PCA will extract at most $m$ principal components, but they will not be representative for all data, or they will fail to retain any relevant detail from it (identifying details is essential for diffraction imaging). Because of computer memory constraints the number of variables cannot be chosen arbitrary big. For example in our investigations we used values up to 200. On the contrary, the number of samples is limited just by the computation power.

For the case of seismic images, in general, a natural way to select similar events is to follow the geological formations, which are depicted in seismic images by horizons. In Figure 4 we show a regular seismic image and many auto-picked horizons by that follow the trend of an identified geological formation. In the PCA method for specularity gathers which we describe here, the variable is specularity, while the samples can be picked from events that correspond to given positions in the undersurface. 


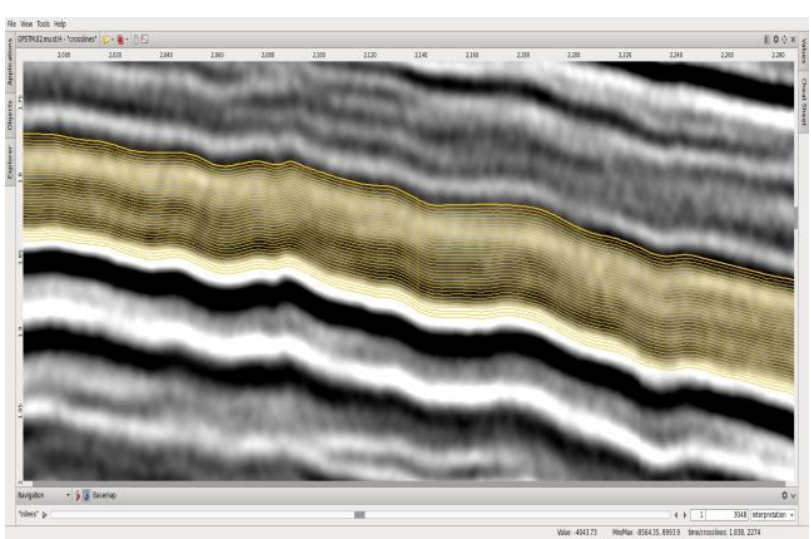

Figure 4: Auto-picked horizons above and below a geological formation.

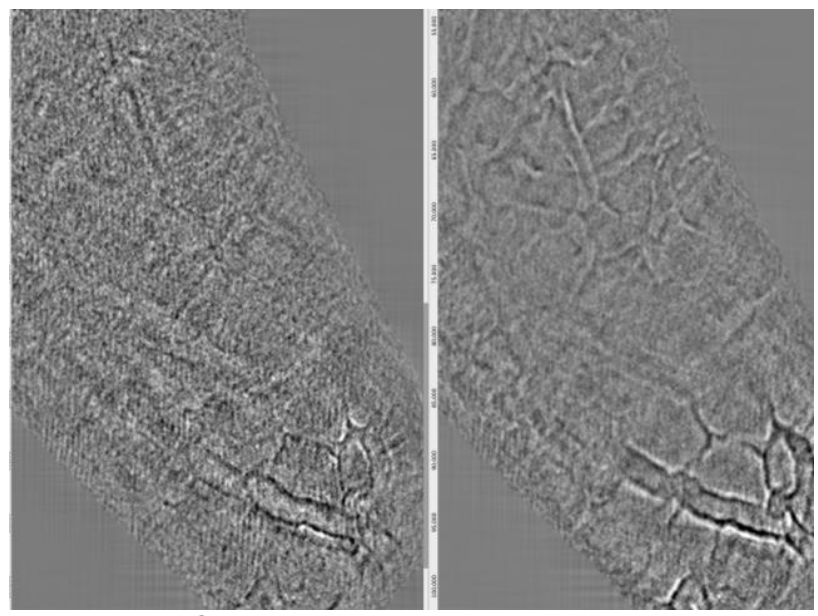

Figure 5: Comparison between a Diffraction Imaging depth slice on the left and a PCA filtered DI depth slice on the right. The filtered DI slice shows higher resolution details of discontinuities.

The depth slices in Figure 5 compare the standard diffraction image with the PCS/SVD filtered DI image. The PCA filtered diffraction images consistently show much higher resolution detail than either the standard DI.

\section{Conclusion}

We discuss the implementation in image domain of Diffraction Imaging, a method of imaging discontinuities in the earth like fractured zones, fault edges, small scale faults, pinch-outs, reef edges, channel edges, salt flanks, reflector unconformities, near surface scattering objects and in general any small scattering objects.

We use Diffraction Imaging as a complement to the structural images produced by reflection imaging. We show results in different areas of the world, in fractured carbonate and unconventional shale reservoirs. We show that operating in a migration framework on pre-stack data, using procedures which complement those used to enhance specular reflections, allows us to obtain higher resolution information, which is lost in conventional procedures. An efficient way to obtain diffraction images is to first separate the migration events according to the value of specularity angle and azimuth, in a similar way to offset or angle azimuth gathers; diffraction images are produced subsequently using post-processing procedures. The high-resolution potential is demonstrated by several case histories in carbonate reservoirs and unconventional shales, which show much more detail than conventional depth migration or coherence. In addition the variation of the diffractions amplitudes with azimuth gives information about the direction of the local stress field, thus allowing reservoir and drilling engineers to optimize the horizontal well trajectories.

We used multidimensional cross-plots in order to create a map of correlations with the wells which were labeled as productive or, respectively, less-productive. We applied this algorithm using clusters of wells, and using multiple attributes. We verified that this set of attributes is not redundant by performing $\mathrm{PCA}$ and verifying that the singular values are all in the same range of values.

\section{References}

Dell S., and Gajewski D., 2011, Common-reflectionsurface-based workflow for diffraction imaging: Geophysics, 76, S187-S195.

Fomel, S., E. Landa, and M. Taner, 2006, Poststack velocity analysis by separation and imaging of seismic diffractions, in SEG Technical Program Expanded Abstracts 2006: SEG, 514, 2559-2563a.

Khaidukov V., Landa E., and Moser T. J., 2004, Diffraction imaging by focusing-defocusing: An outlook on seismic superresolution: Geophysics, 69, 1478-1490.

Koren Z., Ravve I. and Levy R., 2010, Specular-diffraction Imaging by Directional Angle Decomposition. 72nd EAGE Conference Barcelona, Extended Abstracts.

Moser T. J., and Howard C. B., 2008, Diffraction imaging in depth: Geophysical Prospecting, 56, 627-641.

Moser, T. J., 2009, Diffraction imaging in subsalt geometries and a new look at the scope of reflectivity, in EAGE Subsalt Imaging Workshop: Focus on Azimuth, Cairo, Egypt, Expanded Abstracts: EAGE.

Popovici, A., T. Moser, and I. Sturzu, 2014, Diffraction imaging delineates small-scale natural fractures: American Oil and Gas Reporter, 57, 89-93.

Taner M.T., Fomel S. and Landa E., 2006, Separation and imaging of seismic diffractions using plane wave decomposition. 76th SEG meeting New Orleans, Expanded Abstracts. 